\title{
SOME RESULTS ON AN EQUIVALENCE RELATION ON THE SET OF CLOSED AND BOUNDED VALUED MULTIFUNCTIONS
}

\author{
S.M. AydoĞan, Sh. Rezapour, F.M. Sakar
}

ABstract. By using the notion of the fixed point set of multi-valued mappings, we introduce an equivalence relation on the set of all closed and bounded valued multifunction on a metric space. By using the notion we provide some related results.

2010 Mathematics Subject Classification: 34A08, 34B16.

Keywords: Fixed point set, Equivalence relation, multi-valued mapping.

\section{INTRODUCTION}

In 1966, Sam Bernard Jr. Nadler finished his Ph.D. thesis on differential analysis in university of Georgia ([2]). Later, he published some works about results of his thesis ([3], [4] and [6]). He interested fixed point theory by starting basic notions of fixed points and contractive mappings ([5], [7] and [8]). In 1969, he started study of fixed points of multivalued contractive mappings ([9]). In 1970, he published his most famous work in this area ([10]). Hereafter, many researchers reviewed common fixed points of different types of multivalued contractions (see for example, [11], [12] and [13]). In this paper, we introduce an equivalence relation on the set of all closed and bounded valued multifunction on a metric space. Also by using the notion, we provide some related results.

Let $X$ be a nonempty set, $\mathcal{P}(X)$ the set of all nonempty subsets of $X, T$ a multi-valued mapping on $X$ into $\mathcal{P}(X)$ and $\mathfrak{F}_{T}$ the fixed point set of $T$, that is, $\mathfrak{F}_{T}=\{x \in X: x \in T x\}$. For a topological space $(Y, \tau)$, we denote the set of all nonempty closed subsets of $Y$ by $P_{c l}(Y)$ and the set of all nonempty closed and bounded subsets of $Y$ by $P_{b, c l}(Y)$ whenever $Y$ is a metric space.

Let $(X, d)$ be a metric space, $x \in X$ and $A, B \subseteq X$. It is well-known that $D(x, A)=\inf _{y \in A} d(x, y), H(A, B)=\max \left\{\sup _{x \in A} D(x, B), \sup _{y \in B} D(y, A)\right\}$ and $\delta(A, B)=\sup \{d(a, b): a \in A, b \in B\}$. Then, $H$ is a metric on closed bounded subsets of $X$ which is called the Hausdorff metric. 
S.M. Aydoğan, Sh. Rezapour, F.M. Sakar - Some Results on a Equivalence ...

\section{MAIN RESUltS}

Let $(X, d)$ be a metric space. Denote by $\mathcal{F}$ the set of all multi-valued mappings on $X$ into $P_{b, c l}(X)$. Define the relation $\sim$ on $\mathcal{F}$ by $F \sim G$ whenever $\mathfrak{F}_{F}=\mathfrak{F}_{G}$ for all $F, G \in \mathcal{F}$. One can check that $\sim$ is an equivalence relation on $\mathcal{F}$. Denote by $\tilde{\mathcal{F}}$ the equivalence classes of $\mathcal{F}$, that is, $\tilde{\mathcal{F}}=\frac{\mathcal{F}}{\sim}=\{\tilde{F}: F \in \mathcal{F}\}$. Also, define $\tilde{d}$ : $\tilde{\mathcal{F}} \times \tilde{\mathcal{F}} \rightarrow[0, \infty)$ by $\tilde{d}(\tilde{F}, \tilde{G})=H\left(\mathfrak{F}_{F}, \mathfrak{F}_{G}\right)$. It is easy to see that $(\tilde{\mathcal{F}}, \tilde{d})$ is metric space. Note that, there is a connection between common fixed points of two multivalued mappings $S$ and $T$ whenever $S \in \tilde{T}$.

Lemma 2.1. Let $(X, d)$ be a metric space, $m \geq 1 c>1$ and $S, T: X \rightarrow P_{b, c l}(X)$ two multi-valued mappings such that $\mathfrak{F}_{S} \neq \emptyset$. Suppose that for each $x \in X$ and $y \in S x$ (or $y \in T x$ ) there exists $z \in T y$ (respectively $z \in S y$ ) such that

$$
d^{3 m}(x, y)-\frac{3}{4 \sqrt[3]{4}} c^{2} d^{2 m}(y, z) d(x, y)-\frac{c^{3}}{8} d^{3 m}(y, z) \geq 0 .
$$

Then $\mathfrak{F}_{T} \neq \emptyset$ and $\tilde{S}=\tilde{T}$.

Proof. Let $u \in \mathfrak{F}_{S}$ and $z \in T u$. By using the relation (1), we get

$$
d^{3}(u, u)-\frac{3}{4 \sqrt[3]{4}} c^{2} d^{2}(u, z) d(u, u)-\frac{c^{3}}{8} d^{3}(u, z) \geq 0 .
$$

Hence, $-\frac{c^{3}}{8} d^{3}(u, z) \geq 0$ and so $d(u, z)=0$. This implies that $z=u$ and so $u \in T u$. Thus, $\mathfrak{F}_{T} \neq \emptyset$ and $\mathfrak{F}_{S} \subset \mathfrak{F}_{T}$. A similar proof shows that $\mathfrak{F}_{T} \subset \mathfrak{F}_{S}$. Therefore, $\tilde{S}=\tilde{T}$.

Let $(X, d)$ be a metric space and $V: X \rightarrow P_{b, c l}(X)$ a multi-valued map. We say that $T$ has the property $(M)$ whenever for each convergent sequence $\left\{x_{n}\right\}_{n \geq 0}$ with $x_{n} \rightarrow x$ and $x_{2 n-1} \in T x_{2 n-2}$ for all $n$ (or $x_{2 n} \in T V x_{2 n-1}$ for all $n$ ) we have $x \in T x$.

Theorem 2.2. Let $(X, d)$ be a complete metric space, $S, T: X \rightarrow P_{b, c l}(X)$ two multi-valued mappings, $m \geq 1$ and $c>1$. Suppose that for each $x \in X$ and $y \in S x$ (or $y \in T x$ ) there exists $z \in T y$ (respectively $z \in S y$ ) such that

$$
d^{3 m}(x, y)-\frac{3}{4 \sqrt[3]{4}} c^{2} d^{2 m}(y, z) d(x, y)-\frac{c^{3}}{8} d^{3 m}(y, z) \geq 0 .
$$

If one of the multi-valued mappings $S$ and $T$ have the property $(M)$, then $\tilde{S}=\tilde{T}$.

Proof. Let $x_{0} \in X$ be an arbitrary element and $x_{1} \in S x_{0}$. Choose $x_{2} \in T x_{1}$ such that $d^{3 m}\left(x_{0}, x_{1}\right)-\frac{3}{4 \sqrt[3]{4}} c^{2} d^{2 m}\left(x_{1}, x_{2}\right) d\left(x_{0}, x_{1}\right)-\frac{c^{3}}{8} d^{3 m}\left(x_{1}, x_{2}\right) \geq 0$. There exists 
S.M. Aydoğan, Sh. Rezapour, F.M. Sakar - Some Results on a Equivalence ...

$x_{3} \in S x_{2}$ such that $d^{3 m} d\left(x_{1}, x_{2}\right)-\frac{3}{4 \sqrt[3]{4}} c^{2} d^{2 m}\left(x_{2}, x_{3}\right) d\left(x_{1}, x_{2}\right)-\frac{c^{3}}{8} d^{3 m}\left(x_{2}, x_{3}\right) \geq 0$. By continuing this process we obtain a sequence $\left\{x_{n}\right\}_{n \geq 0}$ in $X$ such that $x_{2 n-1} \in S x_{2 n-1}$ and $x_{2 n} \in T x_{2 n-1}$ for all $n$ and

$$
d^{3 m}\left(x_{n}, x_{n-1}\right)-\frac{3}{4 \sqrt[3]{4}} c^{2} d^{2 m}\left(x_{n}, x_{n+1}\right) d\left(x_{n}, x_{n-1}\right)-\frac{c^{3}}{8} d^{3 m}\left(x_{n}, x_{n+1}\right) \geq 0
$$

for all $n$. Note that, the inequality (2) is a third degree polynomial in the variable $d^{m}\left(x_{n}, x_{n-1}\right)$ with the discriminant

$$
\Delta=4\left(\frac{-3}{4 \sqrt[3]{4}} c^{2} d^{2 m}\left(x_{n}, x_{n+1}\right)\right)^{3}+27\left(\frac{-c^{3}}{8} d^{3 m}\left(x_{n}, x_{n+1}\right)\right)^{2} .
$$

Thus, $d^{m}\left(x_{n}, x_{n-1}\right) \geq-2 \sqrt[3]{\frac{c^{3}}{8} d^{3 m}\left(x_{n}, x_{n+1}\right)}=c d^{m}\left(x_{n}, x_{n+1}\right)$. If $k^{m}=\frac{1}{c}$, then we obtain $k<1$ and $0 \leq d^{m}\left(x_{n}, x_{n+1}\right)<k^{m} d^{m}\left(x_{n}, x_{n-1}\right)$. This implies that $d\left(x_{n}, x_{n+1}\right) \leq k d\left(x_{n-1}, x_{n}\right)$ for all $n$. Hence, $d\left(x_{n}, x_{n+1}\right) \leq k^{n} d\left(x_{0}, x_{1}\right)$ for all $n$. It is easy to see that $d\left(x_{n}, x_{n+p}\right) \leq \frac{k^{n}}{1-k} d\left(x_{0}, x_{1}\right)$ for all $n$ and $p$. Thus, $\left\{x_{n}\right\}_{n \geq 0}$ is a Cauchy sequence in $X$. Choose $u \in X$ such that $x_{n} \rightarrow u$. Since $x_{2 n-1} \in S x_{2 n-1}$ and $x_{2 n} \in T x_{2 n-1}$ for all $n$ and one of the multi-valued mappings $S$ and $T$ have the property $(M)$, we conclude that $u \in S u$ or $u \in T u$. By using Lemma 2.1, we get $\tilde{S}=\tilde{T}$.

We need the followings for our last result.

Lemma 2.3. [13] Let $(X, d)$ be a metric space, $A$ and $B$ two bounded subsets of $X$ and $k>1$. Then for each $a \in A$ there exists $b \in B$ such that $d(a, b) \leq k H(A, B)$.

This implies easily next Lemma.

Lemma 2.4. [13] Let $(X, d)$ be a metric space, $k>1$ and $S, T: X \rightarrow P_{c l, b}(X)$ two multi-valued mappings. Then for each $y \in S x$ (or $y \in T x$ ) there exists $z \in T y$ (respectively $z \in S y)$ such that $d(y, z) \leq k H(S x, T y)$.

Theorem 2.5. Let $(X, d)$ be a complete metric space, $T_{1}, T_{2}: X \rightarrow P_{b, c l}(X)$ two multi-valued mappings, $m \geq 1$ and $c>1$. Suppose that for each $x, y \in X$ with $c^{2} \delta^{2 m}\left(y, T_{2} y\right)+6 c \delta^{m}\left(y, T_{2} y\right) \delta^{m}\left(x, T_{1} x\right)+8 \delta^{2 m}\left(x, T_{1} x\right) \neq 0$ we have

$$
H^{m}\left(T_{1} x, T_{2} y\right) \leq \frac{8 d^{3 m}\left(x, T_{1} x\right)}{c^{2} \delta^{2 m}\left(y, T_{2} y\right)+6 c \delta^{m}\left(y, T_{2} y\right) \delta^{m}\left(x, T_{1} x\right)+8 \delta^{2 m}\left(x, T_{1} x\right)} .
$$

Then $\tilde{T}_{1}=\tilde{T}_{2}$. 
S.M. Aydoğan, Sh. Rezapour, F.M. Sakar - Some Results on a Equivalence ...

Proof. By using the inequality (3), we obtain

$H^{m}\left(T_{1} x, T_{2} y\right)\left(c^{2} \delta^{2 m}\left(y, T_{2} y\right)+6 c \delta^{m}\left(y, T_{2} y\right) \delta^{m}\left(x, T_{1} x\right)+8 \delta^{2 m}\left(x, T_{1} x\right)\right) \leq 8 d^{3 m}\left(x, T_{1} x\right)$

for all $x \in X$ and $y \in T_{1} x$. Let $1<c<k^{m}, x \in X$ and $y \in T_{1} x$. By using Lemma 2.4 , there exists $z \in T_{2} y$ such that $d(y, z) \leq k H\left(T_{1} x, T_{2} y\right)$. Hence,

$$
c d^{m}(y, z)\left(c^{2} d^{2 m}(y, z)+\frac{6 c d^{m}}{\sqrt[3]{4}} d^{m}(y, z) d(x, y) \leq 8 d^{3 m}(x, y)\right.
$$

Thus for each $x \in X$ and $y \in T_{1} x$ there exists $z \in T_{2} y$ such that

$$
d^{3 m}(x, y)-\frac{3}{4 \sqrt[3]{4}} c d^{m}(y, z) d^{m}(x, y)-\frac{c^{3}}{8} d^{3}(y, z) \geq 0 .
$$

Now, we show that $T_{1}$ has the property $(M)$. Let $\left(x_{n}\right)_{n \geq 0}$ be a convergent sequence in $X$ with $x_{n} \rightarrow x, x_{2 n-1} \in T_{1} x_{2 n-2}$ and $x_{2 n} \in T_{2} x_{2 n-1}$ for all $n$. Then, we have

$$
d\left(T_{1} x, x_{2 n}\right) \leq H\left(T_{1} x, T_{2} x_{2 n-1}\right)
$$

for all $n$. Hence,

$$
\begin{aligned}
c d^{m}\left(T_{1} x, x_{2 n}\right)\left(c^{2} d^{2 m}\left(x_{2 n-1}, x_{2 n}\right)\right. & \left.\left.+6 c d^{m}\left(x_{2 n-1}, x_{2 n}\right) d^{m}\left(x_{2 n}, T_{1} x\right)+8 d^{(} x_{2 n}, T_{1} x\right)\right) \\
\leq & 8 d^{3 m}\left(x_{2 n}, T_{1} x\right)
\end{aligned}
$$

for all $n$ and so $d\left(x, T_{1} x\right) \leq \frac{1}{c} d\left(x, T_{1} x\right)$, that is, $d\left(T_{1} x, x\right)=0$. Since $T_{1} x$ is a closed subset of $X$, we conclude that $x \in T_{1} x$. Now by using Lemma 2.1 and Theorem 2.2, we get $\mathfrak{F}_{T_{1}}=\mathfrak{F}_{T_{2}}$ and $\tilde{T}_{1}=\tilde{T}_{2}$.

\section{REFERENCES}

[1] R. Bala, B. Ram, Trigonometric series with semi-convex coefficients, Tamang J. Math. 18, 1 (1987), 75-84.

[2] Sam Bernard Jr. Nadler, Some results in differential analysis, Ph.D. Thesis University of Georgia, Ann Arbor, MI (1966).

[3] Sam Bernard Jr. Nadler, A characterization of the differentiable sub-manifolds of $\mathbb{R}^{n}$, Proc. Amer. Math. Soc. 17 (1966), 1350-1352.

[4] Sam Bernard Jr. Nadler, Coverings by closed sets, Portugal Math. 26 (1967), 51-61.

[5] Sam Bernard Jr. Nadler, Horizontally- and vertically-fixed points, J. Natur. Sci. and Math. 7 (1967), 181-189. 
S.M. Aydoğan, Sh. Rezapour, F.M. Sakar - Some Results on a Equivalence ...

[6] Sam Bernard Jr. Nadler, Differentiable retractions in Banach spaces, Tohoku Math. J. 19, 2 (1967), 400-405.

[7] Sam Bernard Jr. Nadler, Sequences of contractions and fixed points, Pacific J. Math. 27 (1968), 579-585.

[8] Sam Bernard Jr. Nadler, Sequences of contractive maps and fixed points, Pacific J. Math. 31 (1969), 659-667.

[9] Sam Bernard Jr. Nadler, Multi-valued contraction mappings, Pacific J. Math. 30 (1969), 475-488.

[10] H. Covitz, Sam Bernard Jr. Nadler, Multi-valued contraction mappings in generalized metric spaces, Israel J. Math. 8 (1970), 5-11.

[11] A. Petcu, Common Fixed Points for Multifunctions Satisfying a Polynomial Inequality, Bul. Univ. Petrol-Gaze Ploiesti Ser. Mat. Inform. Fiz. 62, 2 (2010), 60-65.

[12] V. Popa, Common fixed points for multifunctions satisfying a rational inequality, Kobe J. Math. 2, 1 (1985), 23-28.

[13] I.A. Rus,Fixed points Theorems for Multi-valued Mappings in Complete Metric spaces, Math. Japon. 20 (1975), Special issue, 21-24.

S. Melike Aydoğan

Department of Mathematics,

Faculty of Science and Letters,

Istanbul Technical University,

Istanbul, Turkey

email: melikeaydogan.itu@gmail.com (corresponding author)

Sh. Rezapour

Department of Mathematics,

Azarbaijan Shahid Madani University,

Tabriz, Iran

email: sh.rezapour@azaruniv.edu

F. Müge Sakar

Department of Business Administration,

Faculty of Economics and Administrative Sciences,

Batman University,

Batman, Turkey

email: mugesakar@hotmail.com 